\title{
THESEN UND ARGUMENTE ZU AKTEURSKAUSALITÄT, POTENZEN, DISPOSITIONEN
}

(1) Handlungen sind Verhaltensepisoden, also Ereignisse. Aber nicht alle Verhaltensepisoden sind Handlungen. Statt „eine Verhaltensepisode“ sage ich im Folgenden auch „ein Verhalten“ und lasse zu (als Grenzfall), dass eine Episode momentan ist. Ereignisse, wiederum, sind Individuen mit intrinsischer zeitlicher Verortung. Sie erstrecken sich über ein bestimmtes zeitliches Intervall (eventuell ein degeneriertes, nur einen Zeitpunkt umfassendes). Sie haben dieses Zeitintervall in sich eingebaut, es ist eine unabdingbare Konstituente von ihnen, gehört essentiell zu ihnen. Es gehört bereits dann unabtrennbar zu ihnen, wenn sie noch bloße Möglichkeiten sind, und auch dann, wenn sie bloße Möglichkeiten bleiben.

(2) Jede Handlung hat einen Akteur. (Aber der Akteur kann auch eine Gruppe sein.)

(3) Handlungen sind also Verhaltensepisoden, die einen Akteur haben (aus (1) und (2)). Aber nicht alle Verhaltensepisoden, die einen Akteur haben, sind Handlungen.

(4) Handlungen sind Verhaltensepisoden, die einen Akteur haben und durch diesen Akteur verursacht sind. Und die Umkehrung gilt ebenfalls: Verhaltensepisoden, die einen Akteur haben und durch diesen Akteur verursacht sind, sind Handlungen. (Ein Ereignis, beispielsweise eine Verhaltensepisode, verursachen heißt dabei: eine hinreichende Ursache für es zu sein.) Hiermit ist ein minimaler, ein rein kausaler Handlungsbegriff bestimmt. Anspruchsvollere Handlungsbegriffe lassen sich durch Hinzunahme weiterer Bedingungen definieren. Die Spezifizierung „Verhaltensepisode, die einen Akteur hat und durch diesen Akteur verursacht ist" gibt dann nur eine notwendige, keine auch hinreichende Bedingung für Handlung an.

(5) Ein Ereignis E, das sowohl durch eine Person $X$ verursacht ist als auch, separat, durch eine von $X$ verschiedene Person $Y$ (ein Fall kausaler Überdetermierung), ist dadurch allein noch keine Handlung von $\mathrm{X}$ oder von $\mathrm{Y}$, also auch nicht von $\mathrm{X}$ und von $\mathrm{Y}$; es ist dadurch allein auch noch keine Handlung von $\mathrm{X}$-und-Y (d.h., keine Handlung dieser Personen- 
gruppe). Um eine Handlung von $\mathrm{X}$ zu sein, muss $\mathrm{E}$ eine Verhaltensepisode mit $\mathrm{X}$ als Akteur sein. Um darüber hinaus auch eine Handlung von $\mathrm{Y}$ zu sein, müsste $E$ zudem eine Verhaltensepisode mit $Y$ als Akteur sein. Nun ist aber nicht nur davon auszugehen, dass jede Verhaltensepisode einen Akteur hat - jedes Verhalten ist ja eo ipso ein Verhalten von jemanden oder von etwas -, sondern auch davon, dass sie nicht mehr als einen Akteur hat: Es scheint unmöglich, dass ein und dasselbe Verhalten - als ganzes - sowohl ein Verhalten von jemandem (von etwas) als auch ein Verhalten von jemand anderem (von etwas anderem) ist. (Der Akteur eines Verhaltens kann allerdings eine Gruppe sein; aber das bedeutete nicht, dass das Verhalten - als ganzes - mehr als einen Akteur hat.) Die Konsequenz ist, dass jede Handlung genau einen Akteur hat (so dass man also immer von dem Akteur einer Handlung sprechen kann); denn jede Handlung ist eine Verhaltensepisode, und jede Verhaltensepisode hat nun eben genau einen Akteur. (Klarerweise ist hier vorausgesetzt, dass ein Akteur einer Handlung immer Akteur der Verhaltensepisode ist, die die Handlung ist; aber diese Voraussetzung ist äußerst plausibel, ja erscheint unbezweifelbar.)

(6) Es ist möglich, Ursache für ein Ereignis zu sein, ohne es zu verursachen; denn Ursachen sind nicht eo ipso hinreichende Ursachen. Es ist auch möglich, ein Ereignis zu verursachen, also hinreichende Ursache für es zu sein, obwohl es nicht eintreten würde, wenn dieser oder jener Umstand nicht gegeben oder aber, im Gegenteil, gegeben wäre; denn hinreichende Ursachen sind nicht eo ipso schon aus sich selbst heraus hinreichende Ursachen (also Ursachen, die auf die Anwesenheit oder Abwesenheit gewisser Umstände nicht angewiesen sind).

(7) Eine befriedigende Handlungstheorie muss eine kausale Handlungstheorie sein. Denn Handlungen sind qua Handlungen - im Gegensatz zu bloßen Verhaltensepisoden - Verhaltensepisoden, für die deren Akteur verantwortlich gemacht werden kann. Es ist dies ein präexplikatives Bedeutungspostulat für den Handlungsbegriff; jede Analyse (oder besser gesagt: Explikation) des Handlungsbegriffs muss es respektieren. Verantwortlichkeit des Akteurs für sein Verhalten gibt es nun aber nur durch die Kausalität des Akteurs. Der Akteur einer Verhaltensepisode kann, erstens, nur dann wenigstens partiell für diese Episode verantwortlich gemacht werden, wenn er eine Ursache für sie ist, wenn auch nicht unbedingt eine hinreichende. Der Akteur einer Verhaltensepisode kann, zweitens, nur dann simpliciter für diese verantwortlich gemacht werden, wenn er eine hinreichende Ursache für sie ist, wenn auch nicht unbedingt eine schon aus sich selbst heraus hinreichende. Drittens und endlich kann der Akteur einer Verhaltensepisode nur dann voll für diese verantwortlich 
gemacht werden, wenn er eine schon aus sich selbst heraus hinreichende Ursache für sie ist. Es ist der Beachtung wert, dass in den drei vorausgehenden Sätzen die Kausalität (genauer gesagt: drei Arten von Kausalität) des Handelnden als notwendige Bedingung für seine Verantwortlichkeit (genauer gesagt: für drei entsprechende Weisen seiner Verantwortlichkeit) aufgewiesen wird (werden). Mehr ist nicht gesagt. Man könnte aus der notwendigen Bedingung eine hinreichende machen und damit eine rein kausale Auffassung der Verantwortlichkeit vertreten. Manche allerdings (nicht eben wenige) nehmen das Vorhandensein alternativer Möglichkeiten für den Handelnden als weitere notwendige Bedingung hinzu. Weitere (auch alternativ zu der letztgenannten Bedingung verwendbare) Kandidaten notwendiger Bedingungen für Verantwortlichkeit stellen ab auf Intention und hinreichendes situatives Wissen. Nichts in der Frage der Definition der Verantwortlichkeit ist an dieser Stelle präjudiziert - außer eben, dass die Kausalität des Handelnden eine notwendige Ingredienz in dieser Definition ist.

(8) Wie aus (7) ersichtlich ist, ist jede befriedigende Handlungstheorie nicht nur eine kausale, sondern, zumindest an der begrifflichen Oberfläche, auch eine akteurskausale. Das bedeutet freilich noch nicht, dass sie auch in der begrifflichen Tiefe eine akteurskausale ist. Sie ist keine in der begrifflichen Tiefe akteurskausale Handlungstheorie, wenn sich die Rede von Akteuren als Ursachen rational weganalysieren lässt, sich, mit anderen Worten, auf die Rede von Ereignissen als Ursachen rational reduzieren lässt.

(9) Ich bin allerdings der Auffassung, dass eine derartige Reduktion unmöglich ist. Jede Reduktion von Akteurskausalität auf Ereigniskausalität muss ja darin bestehen, dass statt des Akteurs eigentlich - d.h.: in letzter Analyse - ein Ereignis Ursache und gegebenenfalls hinreichende Ursache ist. Man sagt dann, dass es nur eine façon de parler sei, dass Akteur $\mathrm{X}$ Ursache von Ereignis $\mathrm{E}$ ist, dass das, was damit eigentlich gemeint sei, dies sei: dass ein gewisses Ereignis $E^{\prime}$, mit dem $X$ in akausaler Weise verbunden ist, Ursache von $\mathrm{E}$ ist. Damit fält aber die Verantwortlichkeit des Akteurs unter den Tisch: Auch seine Verantwortlichkeit ist nur eine façon de parler. Denn verantwortlich - sei es, wenigstens partiell verantwortlich, simpliciter verantwortlich, oder voll verantwortlich - für eine in Frage kommende Verhaltensepisode ist ja nun, gemäß der vorgeschlagenen Analyse, eigentlich nicht ihr Akteur $\mathrm{X}$, sondern ein gewisses Ereignis $E^{\prime}$, mit dem $X$ in akausaler Weise verbunden ist und das Ursache, oder hinreichende Ursache, oder gar schon aus sich selbst heraus hinreichende Ursache jener Verhaltensepisode ist. Es ist dann auch nur eine façon de parler, dass eine Verhaltensepisode eine Handlung ihres Ak- 
teurs ist. Damit dies mehr als eine façon de parler wäre, müsste ja wirklich der Akteur der Verhaltensepisode sie verursachen, hinreichende Ursache für sie sein, und nicht stattdessen ein Ereignis, mit dem der Akteur der Verhaltensepisode in akausaler Weise verbunden ist. Eine eigentliche Handlung darf, qua eigentliche Handlung, ihrem Akteur in letzter Analyse nicht zustoßen, nicht ihm geschehen. Jede Verhaltensepisode würde nun aber ihrem Akteur in letzter Analyse zustoßen, ihm geschehen, und nicht durch ihn geschehen, wenn Akteurskausalität auf Ereigniskausalität reduzierbar wäre. Wenn also Akteurskausalität auf Ereigniskausalität reduzierbar wäre, gäbe es keine eigentlichen Handlungen. Davon, dass es eigentliche Handlungen gibt, gehe ich aber aus, also davon, dass es Verhaltensepisoden gibt, die durch ihren Akteur verursacht sind, und eben nicht stattdessen verursacht sind durch ein Ereignis, mit dem dieser Akteur in akausaler Weise verbunden ist.

(10) Ich gehe also auch davon aus, dass es eigentliche Akteurskausalität gibt - also Akteurskausalität, die nicht auf Ereigniskausalität reduzierbar ist. Wie aber ist derartige Akteurskausalität möglich? Ich werde einiges dazu sagen, weise hier, an dieser Stelle, nur vorsorglich darauf hin, dass daraus, dass man nicht weiß, wie etwas möglich ist, ja nicht einmal versteht, was es sein soll, nicht folgt, dass es nicht möglich ist, und auch nicht einmal folgt, dass es nicht existiert. Leider werden derartige Fehlschlüsse in der Philosophie der Vergangenheit und Gegenwart nicht selten begangen, ganz zu schweigen davon, dass Philosophen mindestens ebenso voreilig sind zu behaupten, sie verstünden Fremdes nicht, wie sie voreilig sind zu meinen, sie verstünden Eigenes gut.

(11) Man könnte allerdings meinen, es sei doch ganz einfach zu verstehen, wie ein Akteur selbst Kausalität entfalten könne, und nicht bloß an seiner Stelle ein Ereignis, mit dem er in akausaler Weise verbunden ist. Ein Akteur verfüge eben über gewisse Vermögen, Fähigkeiten, Potenzen; die betätige er eben, und wenn er das tut, dann verursacht er eine Verhaltensepisode von sich, und von niemand anderem, handelt also. ' Dazu ist zu sagen: Ich habe nichts gegen passive oder aktive Vermögen, Fähigkeiten, Potenzen; ohne aktive Vermögen könnte ein Akteur keine Kausalität entfalten. Es stellen sich Fragen allgemeiner ontologischer Art, u.a. auch die Frage, ob es sich bei Vermögen, Fähigkeiten, Po-

\footnotetext{
' Die Handlung ist die verursachte Verhaltensepisode. Man könnte aber auch in dem Verursachen selbst eine Handlung sehen. Doch diese Handlung wäre nun gerade kein Ereignis, keine Verhaltensepisode. Wenn man will, kann man innenbleibende Handlung (das Venursachen) und manifeste Handlung (die verursachte Verhaltensepisode) unterscheiden, wobei das Wort „Handlung“ simpliciter immer das Letztere meint.
} 
tenzen um Eigenschaften handelt, die auf Eigenschaften anderer Art, nämlich nichtmodaler Art, reduzierbar sind, oder aber nicht. Doch erscheint mir die Beantwortung derartiger Fragen jedenfalls für die Frage, wie Akteurskausalität möglich ist, zweitrangig. Eine dafür entscheidende Frage ist vielmehr, welche aktiven Vermögen, Fähigkeiten, Potenzen es denn sind, die für Akteurskausalität bedeutsam sind, und was es denn heißt, dass ein Akteur in der Ausübung von Akteurskausalität ein gewisses aktives Vermögen von sich betätige.

(12) Was es nicht heißen kann - darauf möchte ich hier an erster Stelle eingehen. Manche Potenzen eines Akteurs sind dispositional. Angenommen, der Akteur X habe die dispositionale Potenz Y; man kann auch einfach sagen: Akteur $\mathrm{X}$ habe die Disposition $\mathrm{Y}$. Das bedeutet: Falls bei $\mathrm{X}$ eines der möglichen Bedingungsereignisse der Disposition $\mathrm{Y}$ eintritt, dann hat das diesem Bedingungsereignis entsprechende mögliche Manifestationsereignis der Disposition $\mathrm{Y}$ eine mehr als fünfzigprozentige objektive Wahrscheinlichkeit, bei $\mathrm{X}$ einzutreten, und falls $\mathrm{Y}$ eine deterministische Disposition sein sollte, sogar eine hundertprozentige solche Wahrscheinlichkeit. Nun trete bei $X$ ein mögliches Bedingungsereignis der Disposition Y ein. Also hat das dementsprechende mögliche Manifestationsereignis von $\mathrm{Y}$ eine mehr als fünfzigprozentige objektive Wahrscheinlichkeit, bei $X$ einzutreten. Nehmen wir an, es trete tatsächlich bei $X$ ein, und nennen wir es, in der Weise, wie es bei $X$ eintritt, „V(X)“ $\left(, V(X)\right.$ “ wie „Verhalten von $\left.X^{“}\right)$. Im geschilderten Fall kann man aber sicherlich nicht davon sprechen, dass der Akteur $X$ die dispositionale Potenz Y betätigt habe, schon gar nicht in der Ausübung von Akteurskausalität. Betätigt wurde Y vielmehr, wenn man so will (also gewissermaßen, nicht im wörtlichen Sinn), durch das eingetretene Bedingungsereignis von $Y$.

(13) Wenn die Disposition Y keine deterministische ist, so ist freilich Raum für eine nichtüberdeterminierende Verursachung des fraglichen, zur Disposition $Y$ gehörigen Manifestationsereignisses $V(X)$ durch $X$. Aber wenn eine solche Verursachung von $V(X)$ durch $X$ nun vorliegen sollte, dann kann man dennoch nicht davon sprechen, dass $X V(X)$ verursacht habe kraft seiner - oder auch nur einer-Betätigung der Potenz Y. Vielmehr ist es, zumindest vorderhand, ganz unklar, welche Potenz von sich $X$ in der Verursachung von $V(X)$ betätigt hat. Es sei denn, man begnügt sich mit der Antwort, dass $X$ in der Verursachung von $V(X)$ eben seine Potenz, V(X) zu verursachen, betätigt hat. Doch dies scheint, prima facie, nichtssagend.

(14) Dieser Eindruck lässt sich gründlich beseitigen, wenn folgender Fall vorliegt. Betrachten wir den physischen Weltverlauf bis zu einem 
gewissen Zeitpunkt $t_{0}$. Bis zum Zeitpunkt $t_{0}$ (aber diesen Zeitpunkt nicht miteingeschlossen) liege der physische Weltverlauf ontisch fest. Die physische Welt evolviere aber in physikalisch indetermin istischer Weise, und das sei auch zu und nach dem Zeitpunkt $t_{0}$ noch so. Das bedeutet: Wie das $\mathbf{t}_{0}$-Anfangssegment (genauer gesagt: das Vor- $t_{0}$-Anfangssegment) des physischen Weltverlaufs zum vollständigen physischen Weltverlauf zu ergänzen ist, wie es insbesondere zum Zeitpunkt $t_{0}$ selbst zu ergänzen ist, ist durch dieses Anfangssegment und durch die Gesetze der physischen Natur nicht festgelegt, sondern es gibt mehrere physico-naturgesetzlich mögliche physische Fortsetzungen jenes Anfangssegments. Die Gesetze der physischen Natur - die Gesetze der wahren vollständigen Physik (von welcher insbesondere Nichtphysiker gerne glauben, dass wir sie schon so gut wie sicher in unserem Erkenntnisbesitz haben) - mögen dann den verschiedenen, von ihnen zugelassenen Fortsetzungen des $t_{0^{-}}$ Anfangssegments des physischen Weltverlaufs noch determinierte Wahrscheinlichkeiten „mit auf den Weg geben“ (was freilich auch unmöglich sein kann: wenn nämlich die Anzahl der Fortsetzungen transfinit ist); es ändert nichts daran, dass durch das Anfangssegment und die (wahren) physikalischen Gesetze allein nicht determiniert ist, welche von diesen möglichen Fortsetzungen wirklich wird.

(15) Angesichts dieser Situation, die durch die moderne Physik alles andere als ausgeschlossen wird, sondern als immer und überall gegeben angesehen werden kann, ist es natürlich eine berechtigte Frage, durch was es bedingt ist, dass der bislang vorliegende physische Weltverlauf zu und nach $t_{0}$ in ganz bestimmter Weise weitergeht. Eine übliche, sehr weitverbreitete Antwort auf diese Frage ist es zu sagen, dass, wie es weitergeht, eigentlich durch nichts bedingt ist. Was durch die Wahrscheinlichkeiten der möglicherweise vielen möglichen Fortsetzungen noch unbestimmt gelassen wird, das werde durch den bloßen Zufall - d.h., eigentlicher gesprochen, durch nichts - bestimmt. Die Dinge sind auf einmal so-und-so zu $t_{0}$ bestimmt, und vielleicht auch schon darüber hinaus ein Stück weit; auf die Frage, warum, kousal, sie so und nicht anders bestimmt sind - auf diese Frage gebe es keine Antwort, außer eben der Antwort die eigentlich keine ist: durch den Zufall.

(16) Es liegt mir fern, dem ontischen Zufall ${ }^{2}$ das Existenzrecht zu bestreiten. Auch er ist ja gewissermaßen ein Akteur, er ist rein formal betrachtet ein Akteur, der Kausalität ausübt. Ja, die Existenz des ontischen

\footnotetext{
${ }^{2}$ Der ontische Zufall ist rein ontologisch zu definieren; epistemische Begriffe gehen in seine Definition nicht ein. Ein (aktuales) Ereignis ist ontisch zufällig genau dann, wenn es keine hinreichende Ursache hat.
} 
Zufalls zeigt, dass die Existenz mindestens eines Akteurs mitsamt der durch ihn ausgeübten Kausalität widerspruchsfrei mit den Gesetzen der (wahren, vollständigen) Physik vereinbar ist. Denn der ontische Zufall ist, wie gesagt, rein formal betrachtet ein Akteur, der Kausalität ausübt: Er bestimmt das Physische, das bisher noch unbestimmt war, und zwar nach gängiger Auffassung sogar ganz allein. Man könnte ihn auch durch eine fiktive Akteurin darstellen, Tyche. Niemand macht gegen den Zufall wie ich für „ontischer Zufall“ im Folgenden (wie schon in (15)) kurz sage - den Energie- oder Impulserhaltungssatz geltend, niemand die - allerdings nicht der Physik, sondern der materialistischen Metaphysik angehörenden - Prinzipien der kausalen Geschlossenheit des Physischen. Ebenso wenig wie gegen den Zufall kann man aber diese Naturgesetze und Prinzipien gegen andere Akteure ins Feld führen. Gleiches Recht für alle!

(17) Die Anerkennung anderer (eigentlicherer) Akteure neben dem Zufall hat für die Erhaltungssätze keine Konsequenzen, da sich die Physik - wenn sie sich der Metaphysik enthält, wie sie es qua Physik soll ganz gleichgültig in der Frage verhält (und auch rational verhalten kann), wie die Determinationslücken, die sie offen lässt, geschlossen werden. Bei den Prinzipien der kausalen Geschlossenheit des Physischen sieht es anders aus; denn diese Prinzipien fordern, dass allein der Zufall die Determinationslücken schließt. Doch diese Forderung ist eben gewissermaßen ungerecht, beruht auf einer willkürlichen metaphysischen Vorentscheidung, nämlich einer vorgefassten Präferenz für das rein Physische und Ereignishafte.

(18) Wie ergibt sich die besagte Forderung aus den Geschlossenheitsprinzipien? Sowohl das Prinzip ,Jedes rein physische Ereignis, das eine hinreichende Ursache hat, hat ein rein physisches Ereignis als hinreichende Ursache“ als auch das logisch stärkere Prinzip „Jede hinreichende Ursache eines rein physischen Ereignisses ist ein rein physisches Ereignis" verlangen, wenn sie mit einem rein physischen Ereignis konfrontiert werden, welches kein rein physisches Ereignis als hinreichende Ursache hat, als rein logische Konsequenz, dass dieses Ereignis überhaupt keine hinreichende Ursache hat - was nun, metaphorisch, auch so ausgedrückt werden kann: der Zufall - Tyche - ist für das fragliche Ereignis „die hinreichende Ursache“. ${ }^{3}$

\footnotetext{
${ }^{3}$ Hier liegt ein ähnlicher metaphorischer Sprachgebrauch vor wie etwa auch dann, wenn man bei dem, was (eigentlich) kein Gewicht hat, sagt, es habe das Gewicht 0. Dic metaphorische (oder rein formale) Redeweise ist nützlich, denn sie trăgt zur Vereinfachung der Beschreibungen bei.
} 
(19) Von normalen, eigentlichen Akteuren unterscheidet sich der Zufall freilich durch seine komplette Blindheit; schon sprichwörtlich ist ja der Zufall blind. Blindheit heißt hier: Von Erkenntnissen und Wahrscheinlichkeitsannahmen, von Präferenzen, Werten und Zwecken lässt sich Tyche in ihrem Spiel, in ihrem „Handeln“, nicht im Mindesten leiten. Sie ist eine Akteurin von höchster epistemischer und volitionaler Gleichgültigkeit. (Man kann sie daher zwar sehr wohl für vieles „verantwortlich“ machen - wegen der von ihr ausgeübten Quasi-Kausalität -, sie aber dafür nun gewiss nicht tadein oder loben.)

(20) Es ist heute orthodoxe Meinung, dass dem puren Zufall neben der Notwendigkeit, die aus den Gesetzen der physischen Natur fließt, eine Rolle in der Bestimmung der physischen Wirklichkeit einzuräumen ist; man ist damit zu dem zurückgekehrt, wovon die Materialisten der Antike bereits überzeugt waren. Alles andere als orthodox ist aber die Auffassung, dass es neben dem Zufall, der sich rein formal als Akteur ansehen lässt, der Kausalität im Physischen ausübt, noch andere, und zwar eigentliche, echte Akteure gibt, die ebenfalls Kausalität im Physischen ausüben - Akteure, die alles andere als blind, ja, teilweise sogar einigermaßen rational sind. Zufall und Notwendigkeit - braucht es denn mehr als diese beiden?

(21) Von unserem menschlichen Selbstverständnis her braucht es sehr wohl mehr als Zufall und Notwendigkeit. Das ist, glaube ich, keine Frage. Und lässt man den Zufall zu, warum dann nicht auch eigentliche Akteure, die Kausalität im Physischen ausüben? Eine Determinationslücke, die die Gesetze der physischen Natur offenlassen und von der man weithin annimmt, sie würde allein durch den Zufall mit bestimmten physischen Aktualereignissen gefüllt, würde dann statt allein durch den Zufall auch durch eine - möglicherweise unabsehbar große - Schar von Kausalität im Physischen ausübenden Akteuren mit solchen Ereignissen gefüllt, und den Zufall kann man, wie hier schon geschehen, rein formal zu diesen Akteuren dazurechnen. (Es liegt mir, wie gesagt, fern, dem ontischen Zufall sein Existenzrecht zu bestreiten.) Eine zeitabhängige Auswahl aus jener Schar von Akteuren - eine genau hinreichende Auswahl, nennen wir sie „die Tätertotalităt-zu- $t_{0}$ “ - bringt dann aus dem, was die physische Vergangenheit im Zusammenspiel mit den Gesetzen der physischen Natur für den Zeitpunkt $t_{0}$ offenlässt, den vollständig bestimmten physischen Weltzustand zu diesem Zeitpunkt als aktualen hervor. Man kann es auch so sagen: Die Tätertotalität-zu- $\mathrm{t}_{0}$ ist ein Gruppenakteur, der das physische 
$\mathrm{t}_{0}$-Weltereignis verursacht; ${ }^{4}$ das physische $\mathrm{t}_{0}$-Weltereignis ist, mit anderen Worten, eine Handlung jener Totalität (denn das physische $\mathbf{t}_{0-}$ Weltereignis kann man gut als das $\mathbf{t}_{0}$-Verhalten der Tätertotalität-zu- $\mathbf{t}_{0}$ ansehen). Jedes Glied und jede (echte) Subgruppe der Tätertotalität-zu- $t_{0}$ verursacht dagegen zwar das physische $\mathrm{t}_{0}$-Weltereignis nicht, ist aber doch eine Ursache - eine Akteursursache unter anderen - jenes Ereignisses. Das kann involvieren, dass Teilereignisse des physischen $\mathbf{t}_{0}$ Weltereignisses von Subgruppen oder gar von Gliedern der Tätertotalität verursacht werden; dass diese Teilereignisse sogar Handlungen jener Subgruppen bzw. Glieder sind - ja, es ist angesichts der Phänomenologie des menschlichen Lebens sehr plausibel davon auszugehen, dass es so ist.

(22) lch komme auf die Frage zurück, mit der wir Abschnitt (13) hinter uns gelassen haben: Welche Potenz von sich hat Akteur $X$ in der Verursachung von $\mathrm{V}(\mathrm{X})$ - des relevanten Verhaltens von $\mathrm{X}$ - betätigf? Wenn die Tätertotalität-zu- $t_{0}$ unser Akteur $X$ ist, dann hat diese Frage eine informative Antwort. $\mathrm{Zu} \mathrm{t}_{0}$ können mehrere physische Weltereignisse eintreten; maximal für jedes dieser Weltereignisse ist dessen Verwirklichung eine $\mathbf{t}_{0}$-Potenz der Tätertotalität, minimal für genau eines. Sei $\mathrm{E}^{*}$ dasjenige Weltereignis - eines der besagten möglichen Weltereignisse -, das die Tätertotalität-zu- $\mathrm{t}_{0}$ verursacht; $\mathrm{E}^{*}$ ist also (es spricht nichts dagegen, es so $\mathrm{zu}$ betrachten) das $\mathbf{t}_{0}$-Verhalten der Tätertotalität, das durch diese verursacht wird und dadurch zu einer Handlung von ihr gemacht wird. Die Tätertotalität-zu- $t_{0}$ hat in der Verursachung von $E^{*}$ zweifellos ihre $t_{0}$ Potenz, $\mathrm{E}^{*}$ zu verursachen, betätigt; diese Potenz ist aber nichts anderes als die $\mathrm{t}_{0}$-Potenz der Tätertotalität, $\mathrm{E}^{*} \mathrm{zu}$ verwirklichen.

(23) Das Verursachen eines Ereignisses durch einen Akteur ist also ein Verwirklichen - ein Wirklichmachen - dieses Ereignisses durch einen Akteur. Kein Ereignis hingegen kann ein Ereignis - sich selbst oder ein anderes - wirklich machen. Ereignisse, auch wenn sie wirkliche sind, sind nicht dazu geeignet, andere Ereignisse wirklich zu machen, sondern nur dazu, für gewisse andere wirkliche Ereignisse eine aus naturgesetzlichen Gründen notwendige Vorgabe zu sein, oder gewisse andere wirkliche Ereignisse nach einer naturgesetzlichen Regel auf sich folgen zu haben, oder das Nachfolgen gewisser anderer wirklicher Ereignisse wenigstens naturgesetzlich wahrscheinlich sein zu lassen. Sieht man Verwirklichen

\footnotetext{
${ }^{4}$ Man beachte: Die Tātertotalităt-zu- $\mathrm{t}_{0}$ kann sehr wohl (muss aber nicht) identisch sein mit der Tătertotalität-zu- $t_{1}$ (wobei $t_{0}$ und $t_{1}$ verschiedene Zeitpunkte sind); das physische $t_{0}$-Weltereignis und das physische $t_{1}$-Weltereignis hingegen können nicht miteinander identisch sein (denn $t_{0}$ bzw. $t_{1}$ gehören jeweils essentiell $z u$ dem entsprechenden Momentanereignis als inneres Konstituens).
} 
als Ursächlichkeit im vollen Sinn an, so erweist sich, dass entgegen der neuzeitlichen Tendenz, Ereigniskausalität als die alleingültige, ausschlieBlich haltbare Kategorialform der Kausalität herauszustellen, Ereignisse nun gerade zur Ursächlichkeit im vollen Sinn gar nicht in der Lage sind.

(24) Die Zeit des Eintretens eines Ereignisses, wenn es eintritt, d.h.: wirklich wird, ist das dem Ereignis intrinsische Zeitintervall, gegebenenfalls der ihm intrinsische Zeitpunkt (vgl. (1)). Wenn das Ereignis hingegen nicht eintritt, dann hat es zwar immer noch ein intrinsisches Zeitintervall, gegebenenfalls einen intrinsischen Zeitpunkt, aber eben keine Zeit des Eintretens. Wird ein Ereignis durch einen Akteur verursacht und durch kein Ereignis, so ist dementsprechend die Zeit der Verursachung oder Verwirklichung des Ereignisses keine andere als die intrinsische Zeit des Ereignisses selbst (man beachte aber, dass die Rede von einer Verursachungszeit nicht dazu verleiten darf, ein Verursachen, eine Verursachung als ein Ereignis anzusehen); ${ }^{5}$ ebenso ist, wenn ein Ereignis durch einen Akteur verursacht wird und durch kein Ereignis, die Zeit der Betätigung der entsprechenden Verursachungspotenz durch den Akteur sowie die Zeit des Habens dieser Potenz keine andere als die intrinsische Zeit des Ereignisses selbst.

(25) Es wird gelegentlich (immer wieder) als grundsätzlicher Einwand gegen die von einem Akteur, nicht von einem Ereignis ausgeübte Kausalität vorgebracht, dass bei ihr die hinreichende Ursache - der Akteur - in der Regel schon lange vor der Zeit der Verursachung vollständig existiert, und in der Regel noch lange danach vollständig weiterexistiert. Warum also tritt die Wirkung nicht schon vor der tatsächlichen Verursachungszeit ein, warum nicht auch noch später als diese? Auf diese Fragen scheint es keine Antworten zu geben, was als Indiz einer schwerwiegenden Mangelhaftigkeit des Begriffs der (irreduziblen) Akteurskausalität aufgefasst wird - aus welcher Mangelhaftigkeit sich dann auch gleich die Nichtexistenz der Akteurskausalität ergeben soll (was freilich ein non sequitur ist). In Entgegnung auf den geschilderten Einwand ist zunächst zu sagen, dass Akteure zwar wie Ereignisse nichtabstrakte Individuen sind, aber - im Unterschied zu Ereignissen - keine intrinsische zeitliche Dimension haben (damit auch keine intrinsische zeitliche Lokalisierung, keine intrinsische zeitliche Ausdehnung, keine zeitlichen Teile). Es ist daher ganz richtig: Wenn Akteure schon lange vor dem verursachten Er-

${ }^{5}$ Das Verursachte muss ein Ereignis sein; das Verursachende kann ein Ereignis sein das Verursachen selbst kann es nicht. 
eignis existieren, so existieren sie zu jedem Zeitpunkt dieser VorExistenz vollständig (jeder Teil, den sie da zu einem Zeitpunkt haben, ist zu diesem selben Zeitpunkt auch prösent), und wenn sie gegebenenfalls noch lange danach weiterexistieren, so existieren sie zu jedem Zeitpunkt dieser Nach-Existenz wiederum vollständig. Deshalb gilt: Wann ein Akteur Kausalität ausübt, hängt nicht davon ab, wann er vollständig gegeben ist (er ist ja immer vollständig gegeben, zu jedem Zeitpunkt seiner Existenz), sondern allein davon, für welche Zeitpunkte seiner Existenz eine Gelegenheit zur Ausübung seiner spezifischen Art von Kausalität gegeben ist - d.h.: für welche Zeitpunkte eine Entscheidungssituation, Wahlsituation vorliegt, die in die Reichweite des Akteurs fällt (als Teil der Entscheidungssituation für die jeweilige Tätertotalität $\mathrm{zu}$ den fraglichen Zeitpunkten) - und ob der Akteur die Gelegenheit auch ergreift.

(26) Illustration: Es ist notwendigerweise der Fall, dass das physische Ereignis $E$ zu $t_{0}$ eintritt oder aber nicht $\left(t_{0}\right.$ ist die intrinsische zeitliche Lokalisierung von E). Es liege jedoch weder bereits fest (aufgrund des vorausgehenden physischen Weltverlaufs und der Gesetze der physischen Natur), dass $E$ zu $t_{0}$ eintritt, noch dass $E$ zu $t_{0}$ nicht eintritt. Es liege vielmehr in der Macht von X, dies zu entscheiden. D.h.: $X$ hat die $t_{0}$-Potenz, $E$ zu verwirklichen; aber auch die $t_{0}$-Potenz, ein Ereignis $E^{\prime}$ zu verwirklichen (ebenfalls mit der intrinsischen zeitlichen Lokalisierung $t_{0}$ ), dessen Eintreten zu $\mathrm{t}_{0}$ mit dem Eintreten von $\mathrm{E}$ zu $\mathrm{t}_{0}$ unvereinbar ist. Wenn $\mathrm{X}$ die to-Potenz, Ereignis E zu verwirklichen, betätigt (so wählt, so entscheidet), dann hat $X$ Kausalität ausgeübt: $X$ hat $E$ verursacht (verwirklicht), und zwar zu keinem anderen Zeitpunkt als dem Zeitpunkt $t_{0}$. Davor kann es nicht sein, dass $X \mathrm{E}$ verursacht, denn dafür ist noch nicht die Gelegenheit; danach kann es nicht sein, dass $X \mathrm{E}$ verursacht, denn dafür ist nicht mehr die Gelegenheit. Es könnte auch sein, dass X E zu to nicht verursacht (also auch simpliciter nicht verursacht), sondern an seiner Stelle $E^{\prime}$. Welches von beiden Ereignissen $X$ verursacht, welche Gelegenheit zum Verursachen $X$ ergreift, liegt einzig im Ermessen von $X,{ }^{6}$ in seiner Souveränität (was nicht bedeutet, dass $\mathrm{X}$ blind entscheidet); es wird nicht dadurch entschieden, dass die Ursache nun endlich in dieser oder aber in jener Gestalt, mit diesem oder aber mit jenem Inhalt, vollständig vorliegt. Denn ein Akteur ist nun einmal kein Ereignis, und Akteurskausalität ist grundsätzlich anders verfasst als Ereigniskausalität.

\footnotetext{
${ }^{6}$ Vielleicht ist es sogar möglich und liegt im Ermessen von $X$, dass $X$ keines der beiden Ereignisse verursacht. Wie es mit der Wirklichkeit von $E$ und $E^{\prime}$ steht wăre dann. wenn $X$ keines der beiden Ereignisse verursacht, von anderer Stelle zu entscheiden. notfalls vom Zufall.
} 
(27) Die Tätertotalität-zu- $t_{0}$ verursacht zum Zeitpunkt $t_{0}$ ein inhaltlich maximales Momentanereignis $\mathrm{E}^{*}$ aus einer Menge von mehreren solchen Momentanereignissen, d.h.: aus dem Alternativenpool-zu- $\mathrm{t}_{0}$. Alle und nur (mit anderen Worten: genau) die inhaltlich maximalen physischen Momentanereignisse im Alternativenpool-zu- $t_{0}$ sind aufgrund der physischen Vergangenheit $\mathrm{zu} \mathrm{t}_{0}$ und der Gesetze der physischen Natur nicht auszuschließen als $\mathrm{zu} \mathrm{t}_{0}$ eintretendes inhaltlich maximales physisches Momentanereignis (mit der intrinsischen Zeit $\mathrm{t}_{0}$ ). Es stellen sich drei Fragen: (I) Hätte die Tätertotalität-zu- $t_{0}$ auch gar kein Ereignis aus dem Alternativenpool-zu- $t_{0}$ verwirklichen können? (II) Hätte die Tätertotalität-zu- $\mathrm{t}_{0}$ auch ein anderes Ereignis aus dem Alternativenpool-zu- $\mathrm{t}_{0}$ als $\mathrm{E}^{*}$ verwirklichen können? (III) War die Tätertotalität in der Verursachung von $\mathrm{E}^{*} \mathrm{zu} \mathrm{t}_{0}$ frei? (Von vornherein ist hingegen klar, dass die Tätertotalität nicht mehrere Ereignisse aus dem Alternativenpool-zu- $t_{0}$ verwirklichen kann; denn die Ereignisse in diesem Pool schließen einander inhaltlich aus.)

(28) Auf die Frage (I) weiß ich außer spekulativen Vermutungen keine Antwort. Es scheint mir nicht logisch ausgeschlossen, dass die Tätertotalität-zu- $\mathrm{t}_{0}$ auch gar kein Ereignis aus dem Alternativenpool-zu- $\mathrm{t}_{0}$ verwirklicht. Aber es mag gerade dies aufgrund der für Tätertotalitäten geltenden „Spielregeln“ ausgeschlossen sein. Es sieht ja doch ganz danach aus, dass es ausgeschlossen ist (insbesondere wenn man den Zufall zu den Akteuren zählt). Dieselben „Spielregeln“ könnten nun allerdings womöglich auch fordem (oder vielmehr objektiv erzwingen), dass die Tätertotalität$\mathrm{zu}^{-\mathrm{t}_{0}}$ gerade $\mathrm{E}^{*}$ aus dem zugehörigen Alternativenpool verwirklicht; wer weiß schon, was die „Spielregeln“ sind? - die man gleichwohl befolgen muss, ob man sie nun kennt oder nicht. Wäre es unter solcher Gegebenheit unbedingt der Fall, dass die Tätertotalität kein anderes Ereignis als $E^{*}$ aus dem $t_{0}$-Alternativenpool hätte verwirklichen können? Es scheint so (aber manche meinen, dass es nur so scheint). Und wenn wir einmal dovon ausgehen, dass die Tätertotalität zwar nicht auch gar kein Ereignis aus dem Alternativenpool hätte verwirklichen können, wohl aber ein anderes Ereignis als $E^{*}$, war dann die Tätertotalität-zu- $t_{0}$ in der Verursachung von $\mathrm{E}^{*} \mathrm{zu} \mathrm{t}_{0}$ frei?

(29) Diese Frage ist zu bejahen, wenn die gerade formulierte Voraussetzung von ihr - das, wovon wir bei ihr einmal ausgehen (ich habe es soeben in (28) ausgesprochen) - beinhaltet, dass die Tätertotalität-zu- $\mathrm{t}_{0}$ für mindestens ein weiteres Ereignis außer $E^{*}$ im zugehörigen Alternativenpool die $t_{0}$-Potenz hatte, jenes Ereignis zu verursachen, zu verwirklichen. Und jene Voraussetzung (also die Annahme, dass die Tätertotalität$\mathrm{zu}^{\mathrm{u}} \mathrm{t}_{0}$ ein anderes Ereignis als $\mathrm{E}^{*}$ aus dem Alternativenpool hätte verwirk- 
lichen können) scheint dies (das Haben mindestens einer weiteren einschlägigen Verwirklichungspotenz) in der Tat zu beinhalten.

(30) Die Frage „Kompatibilismus oder Inkompatibilismus?“ hinsichtlich der Freiheit der Tätertotalität-zu- $\mathrm{t}_{0}$ stellt sich nun so: Ist es für diese Freiheit schon hinreichend, wenn, (a), die Tätertotalität ein anderes Ereignis als (das von ihr verwirklichte) $\mathrm{E}^{*}$ aus dem $\mathrm{t}_{0}$-Alternativenpool hätte verwirklichen können (Kompatibilismus)? Oder ist es für diese Freiheit erst hinreichend, wenn, (b), die Tätertotalität für mindestens ein weiteres Ereignis außer (dem von ihr verwirklichten) $E^{*}$ aus dem $t_{0^{-}}$ Alternativenpool die $t_{0}$-Potenz hatte, jenes zu verwirklichen (Inkompatibilismus)? Wenn diese beiden Kandidaten - (a) und (b), d.h., die Kandidaten dafür, die freiheitshinreichende Bedingung zu sein - jedoch so aufgefasst werden, dass (a) (b) logisch beinhaltet, dann entfältt der Sinn der Kompatibilismus-Inkompatibilismus-Frage und damit sie selbst. Eine andere Frage freilich ist zuerst zu beantworten: Können und sollten jene beiden Kandidatenbedingungen so aufgefasst werden, dass die erste die zweite beinhaltet? Ich meine, ja.

(31) Andere (und gewiss manche Leser) sind anderer Auffassung und sehen (a) als oftmals gegebene, bereits für sich genommen freiheitshinreichende Bedingung an - eben auch in den Fällen, wo (b) nicht gegeben und zu verneinen ist. Sie verstehen dabei (a) in einem implizit konditionalen Sinn: Die $\mathrm{t}_{0}$-Tätertotalität hätte klarerweise, sagen sie, ein anderes Ereignis als $E^{*}$ aus dem Alternativenpool-zu- $t_{0}$ verwirklichen können denn das heiße ja nichts anderes als dies: sie hätte ein anderes Ereignis als $\mathrm{E}^{*}$ aus dem Alternativenpool verwirklicht, wenn das und das (anderes als in Wirklichkeit) der Fall gewesen wäre. Es besteht jedoch kein hinreichender Grund, (a) (entgegen der üblichen Auffassung) so zu verstehen. Es besteht insbesondere kein hinreichender Grund, (a), so verstanden, als hinreichend für Freiheit anzusehen, nicht einmal für Freiheit in einem minimalen Sinn. Andere sind anderer Auffassung: Wer mehr als (a) im konditionalen Verständnis für Freiheit verlange (etwa (b), oder (a) in einem solchen Verständnis, dass es (b) beinhaltet), habe dazu keinen hinreichenden Grund. - Das ist nun offenbar ein unauflöslicher begrifflicher Konflikt, wozu auch ein Streit darüber gehört, wer hier eigentlich die Beweislast hat.

(32) Des Fragens ist kein Ende, und so fragt man: Was aber hat dann die Tätertotalität dazu veranlasst, gerade die eine Potenz von sich zu betätigen und nicht die andere? Will man hier nicht in einen infiniten Regress geraten bzw. Akteurskausalität schließlich doch noch auf etwas anderes reduzieren, so muss die Antwort auf diese Frage lauten: Nichts. Die Tätertotalität ist selbst der Ursprung ihrer Verwirklichungswahl. Womit 
man sich sofort die nächste Frage einhandelt: Was aber unterscheidet dann die Tätertotalität-zu- $\mathrm{t}_{0}$ vom Zufall-zu- $\mathrm{t}_{0}$ ? Nun, in einer gewissen Hinsicht muss die Antwort wiederum „Nichts“ lauten: Das Wirken der Tätertotalität-zu- $\mathbf{t}_{0}$ ist nicht weiter kausal zurückführbar, ebenso wie das „Wirken“ des Zufalls nicht weiter kausal zurückführbar ist.

(33) Aber es gibt eben nicht bloß diese eine - die kausale - Hinsicht. So wie der Zufall formal als ein Akteur behandelt werden kann, der Kausalität im Bereich des Physischen ausübt, so kann ein echter Akteur formal als ein Zufallsgenerator für den Bereich des Physischen betrachtet werden. Das Wort „formal" deutet darauf hin, dass man hier um der Vereinheitlichung willen etwas außer Betracht lässt. Was ist es, was man außer Betracht lässt? Wenn man so tut, als wäre der Zufall ein Akteur, dann lässt man außer Betracht, dass der Zufall - anders als ein echter Akteur - nicht im Hinblick auf Ziele agieren kann: Man kann mit dem Zufall keine Intentionen verbinden. Wenn man andererseits so tut, als wäre ein echter Akteur ein Zufallsgenerator, dann lässt man außer Betracht, dass ein echter Akteur - anders als der Zufall - im Hinblick auf Ziele agieren kann: Man kann mit einem echten Akteur Intentionen verbinden. Insofern ist ersichtlich, dass die Tätertotalität-zu- $\mathrm{t}_{0}$ und der Zufall-zu- $\mathrm{t}_{0}$ sehr verschieden sind (mag man auch den Letzteren rein formal zur Ersteren dazurechnen).

(34) Intentionen jedoch (aktuale, nicht dispositionelle) leben in Bewusstseinen, und nirgendwo sonst, als Intentionen von Bewusstseinssubjekten, die zu jedem Zeitpunkt ihres jeweiligen Bewusstseinsstroms als dieselben vollständig anwesend und in ihrem Bewusstseinsstrom - in jedem Erlebnis, in jeder Erfahrung - stets impliziert sind. Das Verhältnis zwischen den Ereignissen, die ein Akteur verursacht, und den Intentionen, die er in seinem Bewusstsein hat, ist dabei ein komplexes. Die Intention zu einem gewissen Zielereignis ist ein Produkt der Präferenzen und Überzeugungen des Akteurs (auch diese Präferenzen und Überzeugungen können wohl bis zu einem gewissen Grad vom Akteur frei gewählt sein). Jene Intention ist dann - of in Konkurrenz $\mathrm{zu}$ anderen Intentionen - Anlass (keineswegs Ursache) für den Akteur, etwas zu verursachen. Tut er dies (wofür auch die Gelegenheit sein muss), dann ist das Ereignis, dessen Eintreten ein Akteur intendiert (in Absehung auf welches er Ursächlichkeit ausübt), gewöhnlich nicht das Ereignis, das der Akteur direkt verursacht (also ohne ereigniskausale Kettenbildung verursacht), und oft auch nicht die Verhaltensepisode von sich, die der Akteur indirekt verursacht (wobei der Akteur als direkte hinreichende Ursache eines Ereignisses fungiert, das am Anfang einer ereigniskausalen Verursachungskette steht, die durch die Verhaltensepisode des Akteurs abgeschlossen wird). 
Dennoch kann man stets davon sprechen, dass der Akteur jene beiden Ereignisse - das direkt verursachte und das indirekt verursachte Verhalten - mit-intendiert (das erstere ohne es überhaupt im Sinn zu haben, das letztere ohne es explizit als intendiert im Sinn zu haben), nämlich als kausale Instrumente zum explizit intendierten (dabei aber oft nur generisch aufgefassten) Zielereignis - ein Zielereignis, von dem dann der Akteur in der Regel tatsächlich nur mehr eine Ursache, aber keine hinreichende Ursache ist, weil an dessen Zustandekommen viele weitere Faktoren beteiligt sind und sein müssen. (Ein Indikator dafür ist, dass viele Zielereignisse nicht erreicht werden, also trotz ausgeübter Akteurskausalität nicht eintreten.)

(35) Zwischen den substanziellen, nichtphysischen, aber im Physischen (nämlich in einem lebenden Organismus) verwurzelten Bewusstseinssubjekten und gewissen echten Einzelakteuren, die als Glieder einer Tätertotalität Kausalität im Physischen ausüben, besteht nach meiner Auffassung Personalunion. Das animalische Bewusstsein und die animalische Akteurskausalität sind im Laufe der Naturgeschichte ko-evolviert und gehören untrennbar zusammen. Denn das animalische Bewusstsein ist die Voraussetzung für eine sinnvolle animalische Akteurskausalität d.h. für eine vom lebendigen Organismus getragene, sehend auf biologischen Vorteil orientierte Akteurskausalität -, sei es im Hinblick auf die niederen, sei es im Hinblick auf die höheren Lebensvollzüge. Die Emergenz der Akteurskausalität cum Bewusstsein - mit identischem Subjekt für Bewusstsein und Aktion - in lebenden Organismen ist nichts anderes als die natürliche evolutive Ausnützung des natürlich gegebenen Indeterminismus der physischen Welt: die Ausnützung einer Chance auf biologischen Vorteil im Kampf ums Überleben. Damit ist nicht unvereinbar, dass die Akteurskausalität und das dazugehörige Bewusstsein sich von der ausschließlich biologischen Bedeutsamkeit auch löst - wie sie es ja beim Menschen offenbar in großem Umfang tut.

(36) Ausführlicher als hier habe ich akteurskausale (oder agenskausale) Theorien des Handelns anderswo behandelt.' Meine Arbeit an diesem

\footnotetext{
${ }^{7}$ Ein Problem ist etwa das Zweite Wie-geht-es-weiter-Problem (wie man es nennen konnte; zum Ersten Wie-geht-es-weiter-Problem. siehe (14) und (15) und die darauffolgenden Abschnitte in diesem Aufsatz). Es kann nicht immer durch Auswahlverwirklichung eines einzelnen, in einem momentanen Weltereignis inkorporierten Weltaustandes weitergehen - es sei denn, die Zeitfolge besteht aus diskreten Positionen. Die $t_{1^{-}}$ Tătertotalität hat gehandelt, und das Vor-to-Anfangssegment des physischen Weltverlaufs ("das $t_{0}$-Anfangssegment“ kann man sagen, wenn man im Gedachtnis behalt. dass $t_{0}$ gerade kein Zeitpunkt dieses Segments ist. sondem ein solcher Zeitpunkt nur allc Zeitpunkte vor $\mathrm{t}_{0}$ sind) ist jetzt um das $\mathrm{t}_{0}$-Weltereignis $\mathrm{E}^{*}$ ergănn. Und nun' Ange-
} 
Thema über eine Spanne von 25 Jahren ist dokumentiert in den Monographien Meixner (1987), (1997), (2001), (2004) und (2010a), daneben in vielen Aufsätzen, beispielsweise in Meixner (2006), (2008), (2010b), (2010c). Dort findet man auch, was man hier vermissen mag: Literaturangaben zu anderen Autoren und die Diskussion der Auffassungen (auch namentlich genannter) anderer Philosophen.

\section{LITERATUR}

Meixner, U. (1987): Handlung, Zeit, Notwendigkeit. Eine ontologischsemantische Untersuchung. Berlin: De Gruyter.

- (1997): Ereignis und Substanz. Die Metaphysik von Realität und Realisation. Paderborn: Schöningh.

- (2001): Theorie der Kausalität. Ein Leitfaden zum Kausalbegriff in zwei Teilen. Paderborn: Mentis.

- (2004): The Two Sides of Being. A Reassessment of Psycho-Physical Dualism. Paderborn: Mentis.

- (2006): Consciousness and Freedom. In: A. Corradini, S. Galvan \& E. J. Lowe (Hrsg.): Analytic Philosophy Without Naturalism. London: Routledge, 183-196.

- (2008): New Perspectives for a Dualistic Conception of Mental Causation. In: Journal of Consciousness Studies 15, 17-38.

- (2010a): Modelling Metaphysics. The Metaphysics of a Model. Heusenstamm bei Frankfurt a. M.: Ontos.

- (2010b): Die Seele als natürliche Instanz der Freiheit. In: K. Crone, R. Schnepf \& J. Stolzenberg (Hrsg.): Über die Seele. Berlin: Suhrkamp, 371-389.

- (2010c): The Emergence of Rational Souls. In: A. Corradini \& T. O'Connor (Hrsg.): Emergence in Science and Philosophy. New York: Routledge, 163-179.

nommen, es ist durch die Gesetze der physischen Natur und durch das, was physisch schon der Fall ist, abermals nicht determiniert, wie es weitergeht. Wenn nun die Zeitfolge aus diskreten Positionen besteht, kann man von $t_{0}$ einfach zum năchsten Zeitpunkt $t_{1}$ öbergehen und die in diesem Aufsatz angestellten Überlegungen, die sich auf $t_{0}$ bezogen, mutatis mutandis auf $\mathrm{t}_{1}$ übertragen. Aber was ist zu tun (in der Theoriebildung), wenn es keinen zu to năchsten Zeitpunkt gibt, wie es der Fall ist, wenn die Zeitfolge dicht oder gar kontimuierlich ist (also wenn die Zeitpunkte aufeinander folgen, wie die rationalen Zahlen aufeinander folgen, oder gar wie die reellen Zahlen)? - Ich lasse diese Frage als offene Frage stehen (eine grundsäzliche Schwierigkeit tut sich hier nicht auf) - neben vielen anderen offenen Fragen, gewiss. 